\title{
Sildenafil ameliorates left ventricular T-tubule remodeling in a pressure overload-induced murine heart failure model
}

\author{
Chun-kai HUANG ${ }^{1,2}$, Bi-yi CHEN², Ang GUO ${ }^{2}$, Rong $\mathrm{CHEN}^{1,2}$, Yan-qi ZHU ${ }^{1,2}$, William KUTSCHKE ${ }^{2}$, Jiang HONG ${ }^{1, *}$, \\ Long-sheng SONG ${ }^{2,3}$ \\ ${ }^{1}$ Shanghai General Hospital, Shanghai Jiao Tong University School of Medicine, Shanghai 200080, China; ${ }^{2}$ Division of Cardiovascular \\ Medicine, Department of Internal Medicine, University of lowa, Carver College of Medicine, lowa City, IA 52242, USA; ${ }^{3}$ Department of \\ Veterans Affairs Medical Center, lowa City, IA 52242, USA
}

Aim: Sildenafil, a phosphodiesterase 5 (PDE5) inhibitor, has been shown to exert beneficial effects in heart failure. The purpose of this study was to test whether sildenafil suppressed transverse-tubule (T-tubule) remodeling in left ventricular (LV) failure and thereby providing the therapeutic benefits.

Methods: A pressure overload-induced murine heart failure model was established in mice by thoracic aortic banding (TAB). One day after TAB, the mice received sildenafil $\left(100 \mathrm{mg} \cdot \mathrm{kg}^{-1} \cdot \mathrm{d}^{-1}, \mathrm{sc}\right)$ or saline for 5 weeks. At the end of treatment, echocardiography was used to examine LV function. Then the intact hearts were dissected out and placed in Langendorff-perfusion chamber for in situ confocal imaging of T-tubule ultrastructure from epicardial myocytes.

Results: TAB surgery resulted in heart failure accompanied by remarkable T-tubule remodeling. Sildenafil treatment significantly attenuated TAB-induced cardiac hypertrophy and congestive heart failure, improved LV contractile function, and preserved T-tubule integrity in LV cardiomyocytes. But sildenafil treatment did not significantly affect the chamber dilation. The integrity of LV T-tubule structure was correlated with cardiac hypertrophy $\left(R^{2}=0.74, P<0.01\right)$ and global LV function $\left(R^{2}=0.47, P<0.01\right)$.

Conclusion: Sildenafil effectively ameliorates LV T-tubule remodeling in TAB mice, revealing a novel mechanism underlying the therapeutic benefits of sildenafil in heart failure.

Keywords: sildenafil; PDE5 inhibitor; T-tubule; pressure overload; left ventricular remodeling; left heart failure

Acta Pharmacologica Sinica (2016) 37: 473-482; doi: 10.1038/aps.2016.13; published online 14 Mar 2016

\section{Introduction}

Heart failure is a common end stage of different cardiovascular diseases and remains a source of considerable morbidity and mortality despite significant advances in its prevention, diagnosis, and treatment ${ }^{[1]}$. In recent years, there has been a tremendous increase in the number of experimental and clinical studies on the use of sildenafil, a phosphodiesterase type 5 (PDE5) inhibitor, for heart failure. Numerous studies have provided evidence that sildenafil delays the progression of heart failure and reverses cardiac remodeling in animal models of heart failure, such as cardiac hypertrophy induced by pressure overload ${ }^{[2-4]}$ and ischemic cardiomyopathy evoked by myocardial infarction ${ }^{[5,6]}$. In clinical studies, sildenafil therapy enhanced exercise tolerance and improved clinical status

\footnotetext{
* To whom correspondence should be addressed. E-mail jhong.pku@163.com

Received 2015-10-31 Accepted 2016-02-06
}

in patients with heart failure and reduced ejection fraction $(\mathrm{HFrEF})^{[7-9]}$; however, unfortunately, sildenafil therapy did not exhibit such beneficial effects in heart failure patients with preserved ejection fraction $(\mathrm{HF} p \mathrm{FF})$ or diastolic heart failure ${ }^{[10,11]}$. A meta-analysis of randomized controlled trials suggests that PDE5 inhibitors have anti-remodeling properties and improve cardiac inotropism independent of afterload changes and with a good safety profile ${ }^{[12]}$. Despite the beneficial effects of sildenafil in heart failure, as demonstrated by basic and clinical research, the mechanism by which sildenafil protects the heart and improves LV pump function remains to be fully defined.

Cardiac transverse-tubules (T-tubules) are regularly spaced invaginations of the surface sarcolemma that occur along the Z-line regions in mammalian ventricular myocytes and branch within the cell to form a highly organized membrane network with predominantly transverse elements but also longitudinal components running from one Z-line to the next ${ }^{[13]}$. T-tubule remodeling has been documented in failing ventricular myo- 
cytes from animal heart failure models of different species and etiologies ${ }^{[14-19]}$, as well as in human heart failure patients with different underlying diseases ${ }^{[16,20]}$. The T-tubule system plays a significant role in cardiac excitation-contraction coupling (E$\mathrm{C}$ coupling) and guarantees the instantaneous excitation and synchronous activation of nearly all $\mathrm{Ca}^{2+}$ release channels/ ryanodine receptors (RyRs) on the sarcoplasmic reticulum $(\mathrm{SR})^{[21]}$. T-tubule remodeling leads to dyssynchronous SR $\mathrm{Ca}^{2+}$ release and defective E-C coupling in heart failure. This is characterized by $\mathrm{Ca}^{2+}$ handling dysfunction, specifically a reduction in $\mathrm{Ca}^{2+}$ release amplitude and slowed kinetics including both time to peak and decay rate of $\mathrm{Ca}^{2+}$ transients, and thus impaired contractility ${ }^{[1,21]}$. Moreover, in failing cardiomyocytes, the structurally remodeled T-tubule system exhibits abnormal electrical activity, i.e., failure of action potential propagation and presence of local spontaneous depolarizations ${ }^{[22]}$. Such T-tubule electrical remodeling is a major determinant of altered kinetics, amplitude, and homogeneity of $\mathrm{Ca}^{2+}$ release. Additionally, spontaneous depolarization in failing T-tubules can trigger local $\mathrm{Ca}^{2+}$ release, resulting in $\mathrm{Ca}^{2+}$ sparks ${ }^{[23,24]}$. Sildenafil treatment applied to well-established cardiac hypertrophy caused by left ventricular (LV) pressure overload can prevent further cardiac and myocyte dysfunction and progressive remodeling, which is associated with improved calcium handling, i.e., enhanced $\mathrm{Ca}^{2+}$ transients and decay $^{[3]}$. We recently reported that sildenafil prevents and reverses T-tubule remodeling and $\mathrm{Ca}^{2+}$ handling dysfunction in right ventricle (RV) failure induced by pulmonary artery hypertension ${ }^{[18]}$. Taken together, these findings lead us to hypothesize that the therapeutic benefit of sildenafil in LV heart failure may include effects at the ultrastructural level, i.e., on T-tubule remodeling.

In the present study, using a thoracic aortic banding (TAB) murine heart failure model, we used in situ confocal imaging of the T-tubule network from Langendorff-perfused intact hearts to test the hypothesis that sildenafil ameliorates T-tubule remodeling following TAB. Here, we demonstrate that early sildenafil administration prevented LV T-tubule remodeling, attenuated cardiac hypertrophy and heart failure progression, and improved LV systolic function under $\mathrm{TAB}$ stress. Therefore, our findings suggest that restoration of T-tubule integrity is one novel mechanism by which sildenafil improves LV function following pressure overload stress.

\section{Materials and methods}

\section{Thoracic aortic banding (TAB) model and sildenafil treatment}

Animal experiments were performed in accordance with the Guide for the Care and Use of Laboratory Animals (National Institutes of Health publication 85-23, revised 1996) and were approved by the Institutional Animal Care and Use Committee at the University of Iowa. Male C57BL/ 6 mice (9-10 weeks of age) were subjected to sham or pressure overload by TAB surgery as described ${ }^{[25]}$. Briefly, mice were anesthetized with ketamine/xylazine (100 mg $\cdot \mathrm{kg}^{-1} / 5 \mathrm{mg} \cdot \mathrm{kg}^{-1}$, ip). The mice were then intubated with a 20 -gauge tube and ventilated using a small rodent ventilator. A thoracotomy was created between the second and third intercostal space, and the aortic arch was visualized. Aortic constriction was performed by tying a 7-0 nylon suture ligature against a 27 -gauge needle to yield a narrowing of $0.4 \mathrm{~mm}$ in diameter when the needle was removed and a reproducible $\mathrm{TAB}$ of $65 \%-70 \%$. In sham mice, the aortic arch was visualized but not banded. Then, the chest wall was closed and the pneumothorax was evacuated. Sildenafil (Pfizer Inc, $100 \mathrm{mg} / \mathrm{kg}$, daily, sc) was administered beginning 1 day after TAB and was continued until 5 weeks after TAB. Control mice were injected with $0.9 \%$ saline. Five weeks after surgery, the numbers of surviving mice for each group were Sham $(n=8)$, TAB $(n=10)$, and TAB+sildenafil (SIL) $(n=8)$. LV function was examined by echocardiography at the end of 5 weeks after sham or TAB. In situ confocal imaging of T-tubule structure from epicardial myocytes of intact hearts was performed on the day after echocardiography.

\section{Echocardiography}

Prior to sacrificing the mice, transthoracic echocardiograms were performed at the University of Iowa Cardiology Animal Phenotyping Core Laboratory using a Vevo 2100 Imager (VisualSonics, Toronto, ON, Canada). Light general anesthesia was achieved with midazolam $(0.2-0.3 \mathrm{mg}$, sc). The anterior chest was shaved, and prewarmed acoustic coupling gel was applied. The 2D images were acquired in the LV short- and long-axis planes with a $30-\mathrm{MHz}$ sector-array probe at a frame rate of 100 frames per second. LV short-axis images were taken at the papillary muscle level and were deemed acceptable when the LV epicardial silhouette best approximated the arc of a circle. LV long-axis views were acquired perpendicular to the short axis and were deemed appropriate when the LV length was longest and both the mitral and aortic valves were contained in the image. The apex was visualized in all mice. Images were stored digitally without analog conversion and were replayed in single-frame mode. End diastole was defined as the frame in which ventricular volume was largest, and end systole was defined as the frame in which ventricular volume was lowest. In the short-axis view, the endocardial and epicardial silhouettes were manually traced at end diastole and end systole by use of the "leading edge-to-leading edge" convention. In the long-axis projection, LV length was measured for both endocardial and epicardial surfaces by measuring from each respective location at the cardiac apex to the center of the mitral valve plane. LV volumes and ejection fraction (EF) were calculated using the biplane arealength method, which has been validated in rodents ${ }^{[26]}$ and humans ${ }^{[27]}$. The 2D-guided LV M-mode images at the level of the papillary muscle were recorded from either the short-axis view or the long-axis view. After completion of 2D imaging, the animals were euthanized, and both the heart and lungs were dissected and weighed.

\section{In situ confocal imaging of myocyte T-tubule structure in intact} hearts

Studies were performed as previously described ${ }^{[17]}$. Briefly, intact mouse hearts were Langendorff-perfused at room tem- 
perature with $\mathrm{Ca}^{2+}$-free Tyrode's solution $(137 \mathrm{mmol} / \mathrm{L} \mathrm{NaCl}$, $5.4 \mathrm{mmol} / \mathrm{L} \mathrm{KCl}, 10 \mathrm{mmol} / \mathrm{L}$ HEPES, $10 \mathrm{mmol} / \mathrm{L}$ glucose, 1 $\mathrm{mmol} / \mathrm{L} \mathrm{MgCl}$, and $0.33 \mathrm{mmol} / \mathrm{L} \mathrm{NaH}_{2} \mathrm{PO}_{4} ; \mathrm{pH}$ adjusted to 7.35-7.45 with $\mathrm{NaOH}$ and oxygenated with $95 \% \mathrm{O}_{2}$ and $5 \%$ $\mathrm{CO}_{2}$ during the experiments) containing $2.5 \mu \mathrm{mol} / \mathrm{L} \mathrm{MM} 4-64$ (AAT Bioquest Inc, Sunnyvale, CA, USA), a lipophilic fluorescence indicator of membrane structure, for $30 \mathrm{~min}$. The hearts were then placed in a perfusion chamber attached to the stage of a confocal microscope and perfused with indicator free $/ \mathrm{Ca}^{2+}$ free solution (with continuous oxygenation). The membrane structure of epicardial myocytes was visualized in situ with confocal microscopy (LSM510, Carl Zeiss MicroImaging Inc, Germany). The microscope was equipped with a $63 \times$ $(\mathrm{NA}=1.4)$ oil-immersion lens. The optical pinhole was set to 1 Airy disc (axial resolution $<1 \mu \mathrm{m}$ ). Ten to twenty T-tubule images were acquired from different locations of LV free walls of each heart. Quantitative analysis of T-tubule integrity was processed offline with custom routines generated using the IDL image analysis program (ITT VIS Inc, Boulder, CO, USA), as previously reported ${ }^{[17]}$. Power values $\left(\mathrm{TT}_{\text {power }}\right)$ from each heart in one group were averaged to represent and reflect the strength of the regularity of the global T-tubule structure of that group.

\section{Criteria for distinguishing murine heart failure}

LV heart failure is characterized by decreased EF and lung edema. Mice with both a significant decrease in EF and an increase in lung weight/body weight $(\mathrm{mg} / \mathrm{g})$ ratio were considered to have heart failure ${ }^{[28]}$. Specifically, in the unbanded control group, values within the range of two standard deviations $(2 \times \mathrm{SD})$ from mean values were considered normal. For $\mathrm{EF}$, the value of $2 \times \mathrm{SD}$ below the unbanded control mean EF (mean $-2 \times \mathrm{SD}=\sim 48 \%$ ) was considered abnormal. For lung weight/body weight ratio, the measure of $2 \times \mathrm{SD}$ above the unbanded control mean lung weight/body weight ratio (mean $+2 \times \mathrm{SD}=\sim 7$ ) was considered abnormal. TAB mice with both an $\mathrm{EF}$ value $<48 \%$ and a lung weight/body weight ratio $>7 \mathrm{mg} / \mathrm{g}$ were classified as having heart failure.

\section{Statistical analysis}

Data were expressed as mean \pm SEM. One-way ANOVA and Student's $t$ tests were used when appropriate. One-way ANOVA was used for multiple group comparisons of in vivo animal experiments. Student's $t$-test was used for two group comparisons. For T-tubule analysis, as multiple images were acquired from each heart in each group, a mixed effects model test was performed to compare the variance found across different hearts to that found within a single heart. The regression/correlation analysis was performed using Winks 4.62 statistics software (TexasSoft, Cedar Hill, Texas, USA). A $P$ value $<0.05$ was considered statistically significant.

\section{Results}

Sildenafil attenuates pressure overload-induced myocardial hypertrophy

We first examined the ability of sildenafil to protect hearts from developing hypertrophy and heart failure under LV pressure overload. The ratios of heart weight to body weight (HW/BW) and lung weight to body weight (LW/BW) for sham-operated mice, TAB mice and TAB mice administered sildenafil beginning 1 day after $T A B$ surgery $(T A B+S I L)$ are illustrated in Figure 1A, 1B. The HW/BW ratio significantly increased from $5.00 \pm 0.14$ in sham-operated mice to $11.81 \pm 0.91$ in TAB mice $(P<0.01)$ at 5 weeks after surgery, which is indicative of cardiac hypertrophy caused by pressure overload. The $\mathrm{LW} / \mathrm{BW}$ ratio also increased more than 2-fold in the $\mathrm{TAB}$ mice compared with the sham-operated mice, suggesting increased fluid volume in the pulmonary vasculature. However, sildenafil significantly attenuated TAB-induced cardiac hypertrophy as shown by changes in the HW/BW ratio from $11.81 \pm 0.91$ to $8.81 \pm 0.66(P<0.05$, Figure $1 \mathrm{~A})$. The $\mathrm{LW} / \mathrm{BW}$ ratio was also significantly reduced with sildenafil interven-
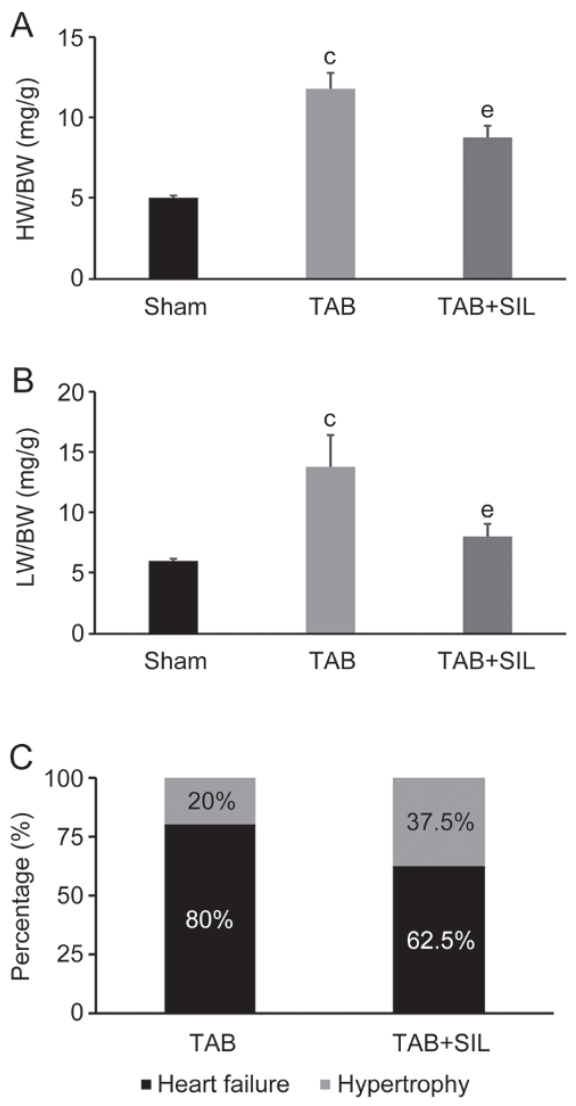

Figure 1. Effects of TAB and sildenafil treatments on cardiopulmonary morphometry. Summary data of heart weight/body weight (A) and lung weight/body weight (B) from unbanded mice, TAB mice 5 weeks after surgery and TAB+SIL mice; $n=8,10,8$ hearts per group, respectively. TAB mice developed pressure overload-induced myocardial hypertrophy and heart failure with significant increases in both HW/BW and LW/ BW. Sildenafil treatment protected against TAB-induced hypertrophy and heart failure, with lower HW/BW and LW/BW in TAB+SIL mice compared with HW/BW and LW/BW in TAB mice. (C) Sildenafil-treated mice are less likely to develop heart failure in response to TAB. $P<0.01$ among groups (A, B), one-way ANOVA. ${ }^{\mathrm{C}} P<0.01$ vs sham. ${ }^{\mathrm{e}} P<0.05$ vs TAB, as indicated, Student's t-test. 
tion $(P<0.05$, Figure $1 \mathrm{~B})$, suggesting that sildenafil prevented pulmonary edema and the development of congestive heart failure in $\mathrm{TAB}$ mice.

We also analyzed the proportion of surviving TAB+SIL mice and $\mathrm{TAB}$ mice with either hypertrophy or heart failure. We previously reported that pulmonary edema was apparent in heart failure but not in hypertrophy and that the LW/BW ratio was significantly increased in failing hearts ${ }^{[17]}$. Therefore, surviving mice with lung weight/body weight ratio $>7(\mathrm{mg} / \mathrm{g})$ and $\mathrm{EF}$ value $<48 \%$ were considered to have developed heart failure (see Methods for details on the definition of heart failure), whereas the other mice were considered to have cardiac hypertrophy. For the TAB group, 8 of 10 surviving mice were classified as having heart failure. Sildenafil decreased the fraction of mice with heart failure to 5 of 8 (Figure 1C). These morphometric data indicate that early sildenafil intervention prevents TAB-induced hypertrophy and the development of heart failure.

\section{Sildenafil improves cardiac function after TAB}

Echocardiography was used to evaluate cardiac function and chamber size to examine how sildenafil affects LV function following TAB. Typical long-axis and short-axis echocardiographic images at end systole and LV M-mode recordings are shown in Figure 2. The baseline long-axis and short-axis views of the sham-operated mice demonstrated normal LV muscle thickness during systole, with typical nearly complete LV emptying in end systole (Figure $2 \mathrm{~A}$, upper left). In contrast, the long-axis and short-axis images of the TAB mice revealed a dilated left ventricular chamber (Figure 2B, middle left). Furthermore, the TAB mice exhibited depressed LV systolic function with a decrease in the reduction in distance between the anterior wall (AW) and the posterior wall (PW) of the LV at end systole (Figure 2B, long-axis view, indicated by arrowheads). Sildenafil failed to significantly reverse the LV dilation induced by pressure overload (Figure 2C, lower left, long-axis and short-axis images). However, the increase in the reduction in distance between the LV anterior and the posterior wall at end systole, compared with that of the TAB group, predicted that sildenafil rescued LV ejection performance impaired by pressure overload (Figure 2C, long-axis view, indicated by arrowheads). The 2D-guided LV M-mode at the papillary muscle level was recorded from either the long-axis view or the short-axis view. Representative LV M-mode echocardiographic recordings showed the dilated heart in the TAB group with significantly increased LV internal dimensions (LVID) at end diastole and systole that was insignificantly rescued in the TAB+SIL mice, consistent with the results of the long-axis and short-axis echocardiographic image data.

Figure 2D-F summarizes the quantitative echocardiographic data from each group. We measured end diastolic volume (EDV), end systolic volume (ESV), and left ventricle ejection fraction (LVEF) following TAB. After 5 weeks of banding, pressure overload damaged LV systolic function and caused ventricular dilatation, consistent with findings from other murine TAB models ${ }^{[4,17]}$. TAB mice displayed dilated LV cavities and enlarged hearts, as demonstrated by massive increases in end systolic and diastolic volumes. Both EDV and ESV were significantly increased in the TAB mice compared with the sham control mice $(99.85 \pm 9.02 \mu \mathrm{L}$ vs $57.46 \pm 4.13 \mu \mathrm{L}$; $68.86 \pm 7.68 \mu \mathrm{L}$ vs $21.40 \pm 2.75 \mu \mathrm{L}$, respectively; $P<0.01$; Figure $2 \mathrm{D}, 2 \mathrm{E})$, paralleling the changes observed in the image data. In addition, a clear decrease in LV contraction was observed in the TAB mice. There was a drastic decline in EF from $63.54 \% \pm 2.70 \%$ in the sham-operated mice to $32.01 \% \pm 2.87 \%$ in the TAB group $(P<0.01$, Figure $2 \mathrm{~F})$, and these findings are suggestive of heart failure.

Sildenafil failed to significantly reverse LV dilation but maintained LV systolic function compared with age-matched $\mathrm{TAB}$ mice (Figure 2D-2F). The quantitative echocardiographic measurements showed that, compared to the TAB group, sildenafil moderately improved EDV and ESV (TAB+SIL vs $\mathrm{TAB}, 93.74 \pm 11.04 \mu \mathrm{L}$ vs $99.85 \pm 9.02 \mu \mathrm{L} ; 52.61 \pm 10.51 \mu \mathrm{L}$ vs $68.86 \pm 7.684 \mu \mathrm{L}$, respectively; $P>0.05$ ), which indicated no rescue of $\mathrm{LV}$ cavity dilation. In contrast, $\mathrm{TAB}+\mathrm{SIL}$ mice showed significantly better $\mathrm{LV}$ contractile function compared with $\mathrm{TAB}$ mice, as shown by increased LVEF $(P<0.05)$. A total of $50 \%$ (4 of 8) of the mice in the sildenafil group had improved LV systolic function (LVEF $>40 \%$ ) after the 5-week sildenafil treatment. Average EF was significantly improved in the TAB+SIL mice compared to that of the control mice in response to TAB stress $(47.67 \% \pm 6.95 \%$ vs $32.01 \% \pm 2.87 \%$, respectively, $P<0.05$, Figure $2 \mathrm{~F}$ ). These echocardiographic data provide evidence that sildenafil improves LV function following TAB.

\section{Sildenafil protects against LV T-tubule remodeling after TAB}

The therapeutic benefit of sildenafil with regard to LV systolic function led us to hypothesize that sildenafil prevents the extensive ultrastructural remodeling in LV myocytes following TAB. T-tubules are one of the key components of cardiac E-C coupling, and disruptive T-tubule remodeling has been documented in many forms of cardiac disease that share the common end-stage of heart failure. To further investigate the subcellular mechanism underlying the beneficial effects of sildenafil on gross cardiac function, we next examined myocyte T-tubule structure using in situ T-tubule imaging. While sham-operated animals displayed a highly organized LV T-tubule network, LV pressure overload caused drastic T-tubule remodeling (Figure 3A, 3B) that corresponded to a severe decrease in $\mathrm{TT}_{\text {power, }}$ an index of T-tubule integrity (Figure 3D). Specifically, at 5 weeks after TAB, the overall LV $\mathrm{TT}_{\text {power }}$ was $1.49 \pm 0.04$ in the TAB mice compared to $2.11 \pm 0.029$ in the sham-operated mice $(P<0.01$, Figure $3 \mathrm{D})$.

As shown in the representative images and quantitated $\mathrm{TT}_{\text {power }}$ data, LV myocytes from the TAB+SIL group had improved T-tubule organization compared to the TAB group (Figure 3C, 3D), suggesting that this ultrastructural alteration is preventable with sildenafil therapy. This improvement of LV T-tubule structure with administration of sildenafil was shown by a significant increase in $\mathrm{LV} \mathrm{TT}_{\text {power }}$ from $1.49 \pm 0.04$ in the TAB group to $1.74 \pm 0.06$ in the TAB+SIL group $(P<0.01$, Figure 3D). 
A

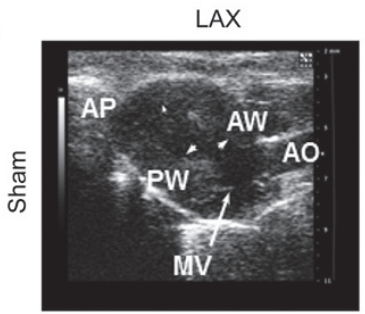

B

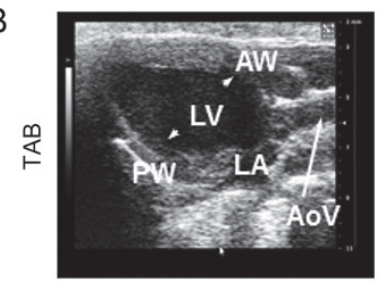

C

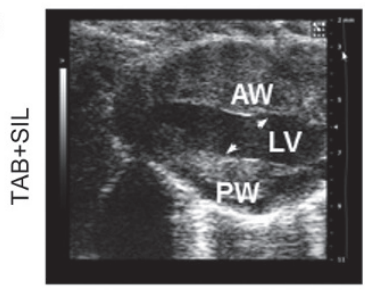

SAX
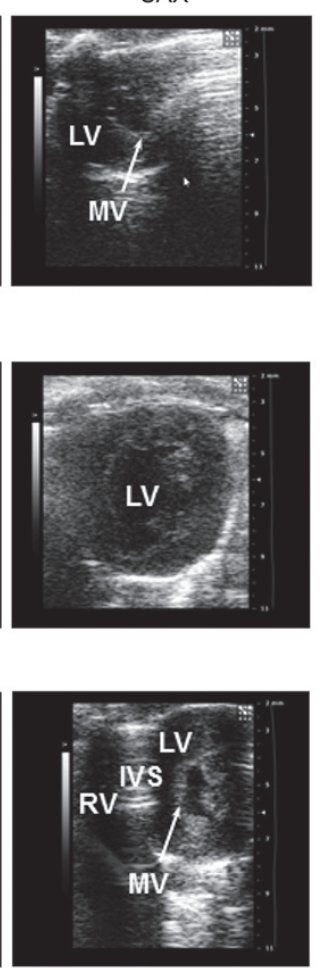

M-mode
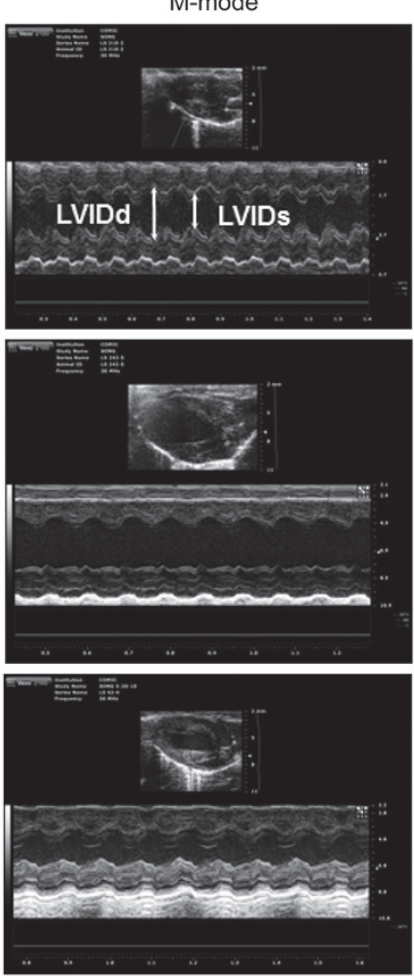

D

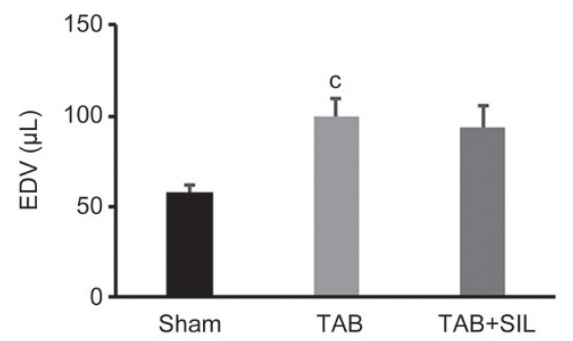

$\mathrm{E}$

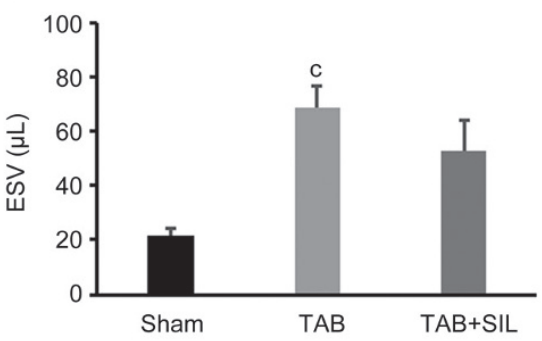

F

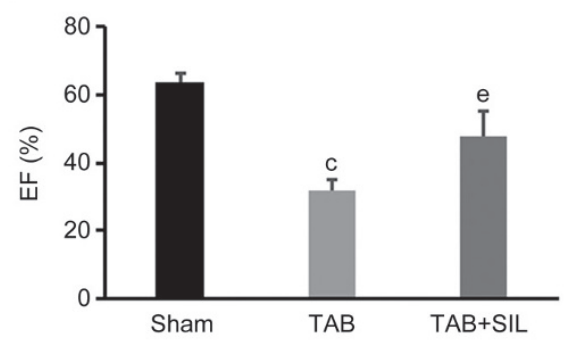

Figure 2. Sildenafil improved cardiac function in TAB mice. (A-C) Representative long-axis and short-axis echocardiographic images at end systole as well as LV M-mode recordings from a sham-operated (top), TAB (middle) and TAB+SIL (bottom) mouse, respectively. In these echocardiographic images, the apex (Ap) of the LV is to the left and the aorta ( $\mathrm{Ao}$ ) is to the right. The mitral valve (MV) and the aortic valve (AoV) are indicated by arrows. Arrowheads point to the endocardial surface of the anterior wall (AW) and the posterior wall (PW) of the LV; reduction in distance between arrowheads indicates contraction of the ventricle during systole. LV internal dimensions (LVID) at diastole and systole (LVIDd and LVIDs, respectively) are measured from M-mode images at the level of the papillary muscles. LA=left atrium, IVS=interventricular septum. (D-E) Echocardiographic measurements of LV function in sham-operated, TAB and TAB+SIL mice. Graphs show mean \pm SEM data for D, end diastolic volume; E, end systolic volume; and F, ejection fraction for each group. LVEF was significantly decreased and LV volume was increased in the TAB mice. These findings indicate that the increase in TAB-induced LV afterload caused LV dilation and impairment of LV systolic function. TAB+SIL mice exhibited enlarged LV chamber size. However, in contrast, treatment with sildenafil normalized LV ejection fraction compared with age-matched TAB mice. $P<0.01$ among groups (D, E, F), oneway ANOVA. ${ }^{\circ} P<0.01$ vs sham. ${ }^{e} P<0.05$ vs TAB, as indicated, Student's $t$-test. $n=8,10$, and 8 per group. EDV, end diastolic volume; ESV, end systolic volume; EF, ejection fraction.

We next analyzed the histogram distribution of $\mathrm{TT}_{\text {power }}$ from individual LV images (Figure 3E). As multiple images were acquired from each heart in each group, we performed a mixed effects model test to compare the variance found across different hearts to that found within a single heart, and we determined that the results were significantly different $(P<0.01)$. Under sham conditions, $\mathrm{TT}_{\text {power }}$ in $\mathrm{LV}$ myocyte populations exhibited a single-mode, bell-shaped distribution (centered at 2.2), suggesting that $\mathrm{TT}_{\text {power }}$ from all LV myocytes belongs to one single population in the unbanded hearts. After TAB stress, the entire histogram of LV TT power $_{\text {shifted }}$ leftward (centered at 1.6). However, the histogram retained its single-mode configuration, suggesting that the entire population of myocytes suffers from T-tubule remodeling in a similar 


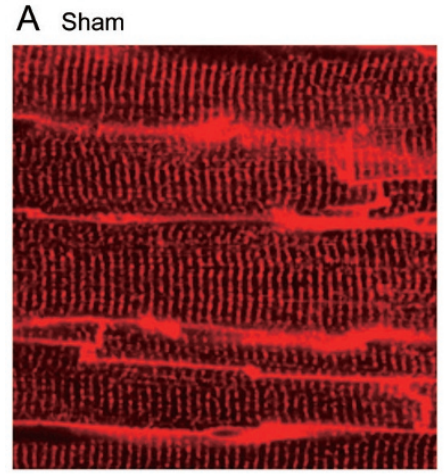

\section{B TAB}
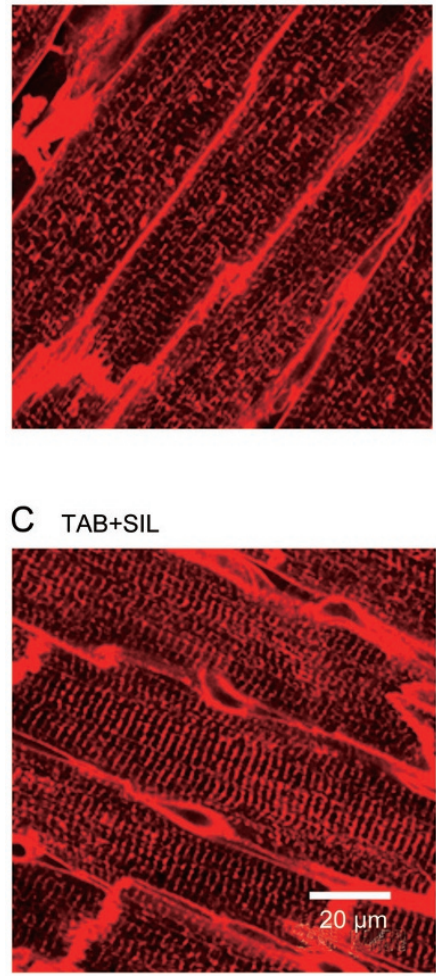
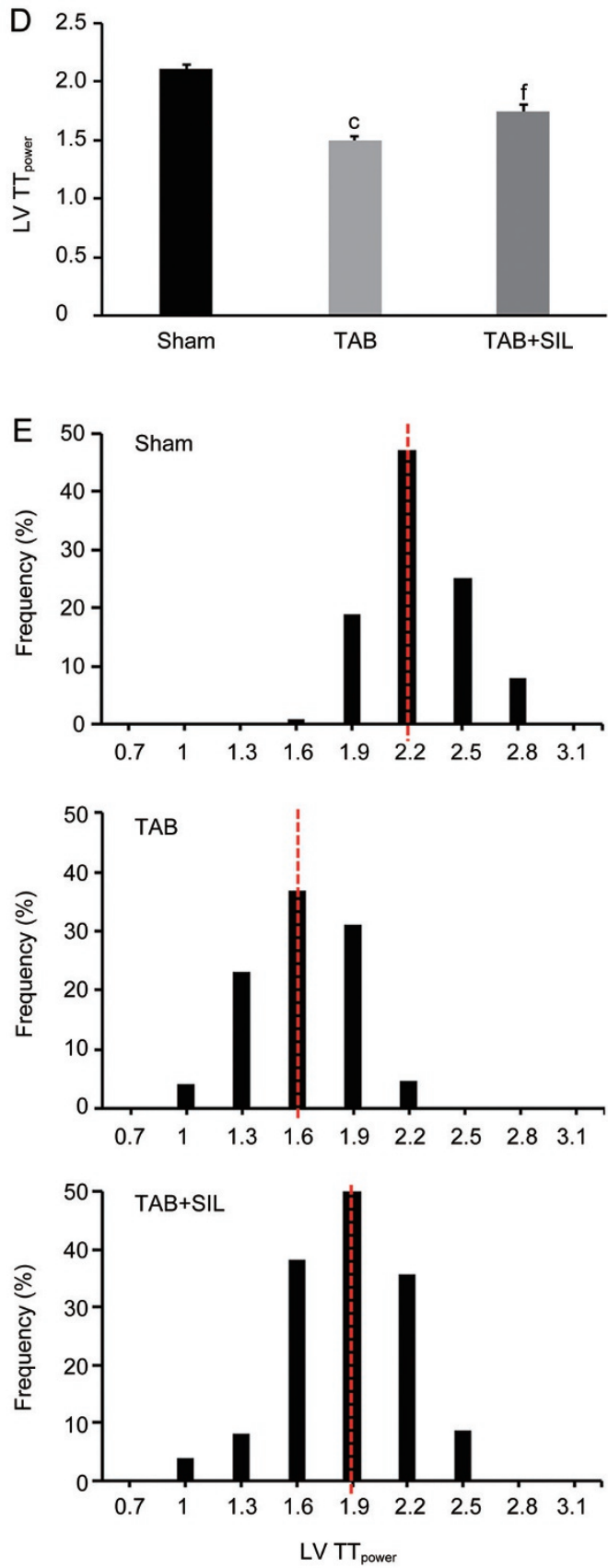

Figure 3. In situ confocal imaging of the LV myocyte T-tubule system from Langendorff-perfused intact hearts. (A-C) Representative LV T-tubule images from the sham, TAB, and TAB+SIL ( $100 \mathrm{mg} / \mathrm{kg}$ sc per day, given 1 day after TAB, for 5 weeks) groups, respectively. (B) TAB-treated mice exhibited severe T-tubule loss and disorganization in LV myocytes. (C) Treatment of sildenafil prevented TAB-induced T-tubule remodeling in LV myocytes. (D) Average data of $L V T_{\text {power }}(T$-tubule power), an index of the strength of T-tubule regularity (for analysis, see Reference 17 ). LV TT power of the TAB+SIL group was significantly higher than that of the TAB group but lower than that of the sham group. (E) Histogram analysis of $\mathrm{TT}_{\text {power }}$ of all $\mathrm{T}$-tubule images acquired from different regions of LV free wall (10-20 images from each LV). The count numbers at each bin size were normalized by the total images of each

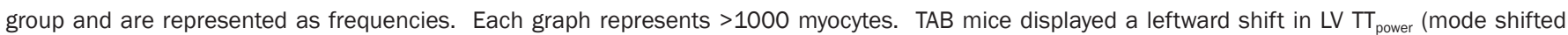
from 2.2 of sham to 1.6). The histogram of the TAB+SIL group was shifted to the right in comparison to that of the TAB group (with a mode shift from 1.6 to 1.9), indicating improvement of LV T-tubule ultrastructure by sildenafil treatment. $P<0.01$, mixed effects model test. $P<0.01$ among groups, one-way ANOVA. ${ }^{\mathrm{C}} P<0.01$ vs sham. ${ }^{\mathrm{f}} P<0.01$ vs TAB. $n=8,10$, and 8 hearts per group.

way. In particular, it is noteworthy that the highest $\mathrm{LV} \mathrm{TT}_{\text {power }}$ $(\geq 2.2)$ was decreased from $33 \%$ in the hearts of sham-operated mice to $0 \%$ in the hearts of TAB mice (Figure 3E, middle).
These data strongly suggest that, in addition to overt subcellular loss of T-tubules in a fraction of myocytes, the entire population of LV epicardial myocytes examined undergoes global 
T-tubule remodeling in response to TAB stress.

For the TAB+SIL group, we found that sildenafil shifted the histograms of $\mathrm{LV} \mathrm{TT}_{\text {power }}$ back to the right in response to $\mathrm{TAB}$, while retaining a single mode of distribution in the LV (centered at 1.9; Figure 3E, bottom), indicating an improvement of LV T-tubule structure. The highest LV TT power $(\geq 2.2)$ was increased from $0 \%$ in the hearts of $\mathrm{TAB}$ mice to $7 \%$ in the hearts of $\mathrm{TAB}+\mathrm{SIL}$ mice. Thus, histogram analysis of $\mathrm{TT}_{\text {power }}$ distribution further confirmed the beneficial effects of sildenafil on TAB-induced LV myocyte T-tubule remodeling.

\section{T-tubule remodeling correlates with LV function}

The role of the T-tubule in cardiac E-C coupling and the consequence of T-tubule remodeling in failing myocytes (ie, SR $\mathrm{Ca}^{2+}$ handling dysfunction) are well recognized ${ }^{[14-16,19,29]}$. Our early study demonstrated that T-tubule integrity is highly correlated with cardiac EF in diseased hearts, indicating that T-tubule integrity is a crucial determinant of cardiac function $^{[17]}$.

Therefore, we examined the correlation between $\mathrm{LV} \mathrm{TT}_{\text {power, }}$ which represents the overall T-tubule status of myocardium, and cardiac morphometrics and performance (Figure 4). LV $\mathrm{TT}_{\text {power }}$ displayed a strong negative correlation $\left(R^{2}=0.74\right.$, $P<0.01$ ) with HW/BW ratio (Figure $4 \mathrm{~A}$ ), a significant index of myocardial hypertrophy. $\mathrm{LV} \mathrm{TT}_{\text {power }}$ also exhibited a positive, linear correlation with LVEF $\left(R^{2}=0.47, P<0.01\right.$; Figure $\left.4 \mathrm{~B}\right)$. These results indicate a structure-function correlation between subcellular T-tubule remodeling and whole-animal cardiac performance in heart failure, further supporting our previous conclusions from a rat pressure overload heart failure
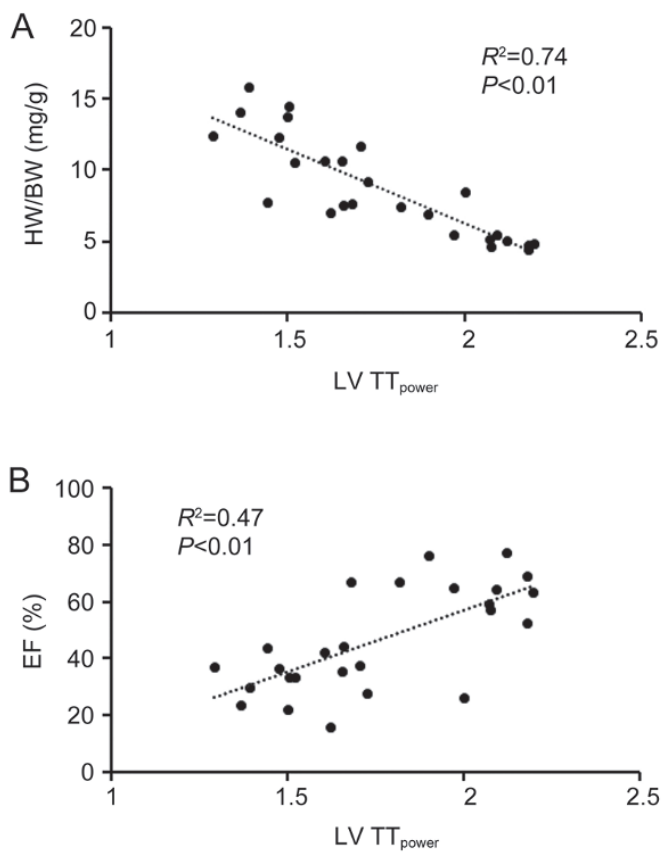

Figure 4. Correlation between $\mathrm{LV} \mathrm{TT}_{\text {power }}$ and $\mathrm{LV}$ function. The $\mathrm{LV} \mathrm{TT}_{\text {power }}$ was correlated with $\mathrm{HW} / \mathrm{BW}$ ratio $\left(R^{2}=0.74, P<0.01\right)(\mathrm{A})$ and $\mathrm{EF}\left(R^{2}=0.47\right.$, $P<0.01)(B)$. $\operatorname{model}^{[17]}$.

\section{Discussion}

Myocardial remodeling and attendant cardiac dysfunction remain major causes of morbidity and mortality in heart failure, and new approaches to combat this pathophysiological process are needed. The therapeutic benefits of sildenafil in heart failure were reported previously, yet the mechanism is still not completely understood. In the present study, using a TAB mouse heart failure model in combination with in situ confocal imaging of myocyte T-tubule structure in intact hearts, we examined the effect of sildenafil therapy on T-tubule remodeling and cardiac function following pressure overload. The major findings of our study are as follows. First, TAB-induced heart failure manifested with marked myocardial hypertrophy, LV dilation, systolic dysfunction, severe T-tubule loss and disorganization in LV myocytes. Second, sildenafil administered at the onset of the TAB stress attenuated the development of cardiac hypertrophy and heart failure, partially restored LV contractile function, and preserved normal T-tubule ultrastructure in LV myocytes. Third, T-tubule structural remodeling was strongly correlated with the degree of hypertrophy and global LV function in this TAB mouse model. These data provide insight into the mechanism by which sildenafil improves cardiac function following TAB.

Compelling experimental and clinical evidence indicates that sildenafil exerts beneficial cardiovascular effects ${ }^{[2-9,12]}$. It has been demonstrated that, at the structural (organ) level, sildenafil prevents and reverses ventricular remodeling in chronic heart failure following pressure overload ${ }^{[2-4]}$. In a mouse model of left ventricular overload by TAB, sildenafil was reported to prevent myocardial hypertrophy, fibrosis, and chamber dilation and to improve in vivo cardiac function ${ }^{[2,4]}$. Additionally, sildenafil reverses pre-established hypertrophy induced by pressure overload while restoring chamber function $^{[4]}$. Further study provided evidence that delayed sildenafil treatment also suppresses progressive cardiac dilation, dysfunction, fibrosis, and hypertrophy in hearts subjected to sustained pressure overload ${ }^{[3]}$. In our experiments, we found that early treatment with sildenafil after TAB prevented afterload increase-induced structural hypertrophy, improved LV contractile function, and thus attenuated the development of heart failure, consistent with the previously published literature ${ }^{[4]}$. However, our findings showed that sildenafil treatment concurrent with $\mathrm{TAB}$ had minimal impact on $\mathrm{LV}$ chamber dilation. Others have suggested that PDE5 inhibition by chronic sildenafil treatment appears to increase the propensity of pressure overload-induced chamber dilation and cardiac failure $^{[30]}$. The mechanism accounting for these mixed results is presently unknown. Chamber dilation in advanced heart failure signifies that ventricular remodeling has reached a point where hypertrophy is no longer adequate to maintain ejection performance. We speculate that sildenafil may lose its protective effects at advanced stages of heart failure and is unable to maintain normal chamber size.

Our data presented in this study extend these observa- 
tions at the whole-organ level and, moreover, demonstrate that sildenafil protects against the ultrastructural subcellular membrane change that accompanies LV failure. Specifically, LV T-tubule remodeling evoked by TAB stress was mitigated with early intervention of sildenafil. We recently reported that sildenafil can prevent and even reverse RV T-tubule remodeling in an RV failure rat model induced by pulmonary artery hypertension ${ }^{[18]}$. However, in this model, the interpretation of sildenafil effects on T-tubule remodeling is complicated by the significant impact of sildenafil on pulmonary artery hypertension. In the present study, we tested whether sildenafil has a direct effect on myocyte T-tubule remodeling following cardiac stress. Our study indeed demonstrated that sildenafil, when applied early after TAB, could prevent pressure overload-induced disruption of the LV myocyte T-tubule system. However, it raises another more clinically applicable question of whether sildenafil can reverse already existing T-tubule disruption. Further investigation is needed to test this by administering sildenafil after heart failure is established following cardiac stress.

Previous work from our group demonstrated that myocardial T-tubule disruptive remodeling is a critical event during the development of hypertrophy and is tightly correlated with the decline of cardiac function. Thus, T-tubule remodeling constitutes a key mechanism underlying the transition from compensated hypertrophy to heart failure, indicating that preventing T-tubule remodeling during the hypertrophy stage may be clinically important for delaying the progression to heart failure ${ }^{[17]}$. Our current data also revealed that LV T-tubule integrity is closely associated with LV contractile function, consistent with our previous results ${ }^{[17]}$. Therefore, we can attribute the improvement in LVEF with sildenafil therapy, at least partly, to the preservation of the T-tubule network. However, many factors contribute to cardiac dysfunction in the progression of heart failure in the TAB model. Accordingly, sildenafil could improve cardiac performance by other mechanisms as well. For example, T-tubule remodeling leads to an increase in orphaned RyRs, a reduction or redistribution in $\mathrm{Ca}^{2+}$ channels on the T-tubule membrane, impaired coupling efficacy between $\mathrm{Ca}^{2+}$ channels on T-tubule and RyRs on the SR, and therefore asynchronous $\mathrm{Ca}^{2+}$ release and a reduction in $\mathrm{Ca}^{2+}$ transient amplitude and kinetics ${ }^{[14,19,31]}$. In line with these findings, it has been reported that isolated myocytes from $\mathrm{TAB}+$ sildenafil hearts show improved calcium cycling $^{[3]}$.

It remains unclear how inhibition of PDE5 with sildenafil mechanistically prevents LV T-tubule remodeling following TAB stress. Though PDE5 is more abundantly expressed in vascular smooth muscle, its expression and activity are increased in human and mouse cardiac hypertrophy and heart failure $^{[4,32,33]}$. We postulate that the anti-T-tubule remodeling effect from selective inhibition of PDE5 by sildenafil may likely be caused by direct cardiac effects. To date, several molecules have been shown to be involved in T-tubule formation and maintenance following cardiac stress, including amphyphisin-2 (Bridging Integrator 1, BIN1) and junc- tophilin-2 (JP2) ${ }^{[17,34-38]}$. BIN1 is downregulated in failing human cardiomyocytes, which impairs Cav1.2 trafficking to T-tubules, calcium transients, and contractility ${ }^{[39]}$. However, no studies have examined the role of BIN1 in T-tubule remodeling in heart failure. Early studies demonstrated an essential role for the Gaq/phospholipase $C \beta$ (PLC $\beta$ ) pathway in the development of compensatory hypertrophy following pressure overload. PDE5 inhibition via sildenafil was found to blunt hypertrophy and enhance cardiac function early after TAB by suppressing Gaq/PLC $\beta$ signaling ${ }^{[40]}$. Recent work from our group using a cardiac-targeted Gaq-overexpressing transgenic mouse model revealed that Gaq/PLC $\beta$-dependent activation of the $\mathrm{Ca}^{2+}$-dependent protease calpain and subsequent downregulation of JP2, a structural protein that bridges the T-tubule and SR membranes, is one molecular mechanism that leads to T-tubule remodeling and heart failure ${ }^{[41]}$. Taken together, these data suggest that sildenafil may effectively prevent T-tubule remodeling through inhibition of this pathway. Future studies using the cardiac-specific Gaq overexpression mouse model are necessary to completely understand the mechanism.

In summary, we elucidated that early intervention with sildenafil preserves LV structure and function at the ultrastructural and whole-organ levels and uncovered a new mechanism by which sildenafil provides therapeutic benefits in heart failure. Specifically, the prevention of T-tubule remodeling appears to be an important benefit of sildenafil in LV pressure overload-induced heart failure. Our study provides new insights into the understanding of sildenafil in heart failure pathogenesis. Further mechanistic investigation would help in reaping additional benefits of sildenafil for heart failure.

\section{Acknowledgements}

This work was supported by the Scientific Research Foundation of Shanghai Jiao Tong University School of Medicine (No 12XJ3008, Jiang HONG), the National Natural Science Foundation of China (№ 81570293, Jiang HONG), and the United States National Heart, Lung and Blood Institute (№ R01 HL090905, Long-sheng SONG).

\section{Author contribution}

Long-sheng SONG and Jiang HONG designed and supervised the research; Chun-kai HUANG, Bi-yi CHEN, and Ang GUO performed the research and analyzed the data; William KUTSCHKE, Rong CHEN, and Yan-qi ZHU assisted with the research; and Chun-kai HUANG, Jiang HONG, and Longsheng SONG wrote the paper.

\section{References}

1 Mudd JO, Kass DA. Tackling heart failure in the twenty-first century. Nature 2008; 451: 919-28.

2 Hsu S, Nagayama T, Koitabashi N, Zhang M, Zhou L, Bedja D, et al. Phosphodiesterase 5 inhibition blocks pressure overload-induced cardiac hypertrophy independent of the calcineurin pathway. Cardiovasc Res 2009; 81: 301-9.

3 Nagayama T, Hsu S, Zhang M, Koitabashi N, Bedja D, Gabrielson KL, et al. Sildenafil stops progressive chamber, cellular, and molecular 
remodeling and improves calcium handling and function in hearts with pre-existing advanced hypertrophy caused by pressure overload. J Am Coll Cardiol 2009; 53: 207-15.

4 Takimoto E, Champion HC, Li M, Belardi D, Ren S, Rodriguez ER, et al. Chronic inhibition of cyclic GMP phosphodiesterase 5A prevents and reverses cardiac hypertrophy. Nat Med 2005; 11: 214-22.

5 Chau VQ, Salloum FN, Hoke NN, Abbate A, Kukreja RC. Mitigation of the progression of heart failure with sildenafil involves inhibition of RhoA/Rho-kinase pathway. Am J Physiol Heart Circ Physiol 2011; 300: H2272-9.

6 Salloum FN, Abbate A, Das A, Houser JE, Mudrick CA, Qureshi IZ, et al. Sildenafil (Viagra) attenuates ischemic cardiomyopathy and improves left ventricular function in mice. Am J Physiol Heart Circ Physiol 2008; 294: H1398-406.

7 Guazzi M, Vicenzi M, Arena R. Phosphodiesterase 5 inhibition with sildenafil reverses exercise oscillatory breathing in chronic heart failure: a long-term cardiopulmonary exercise testing placebocontrolled study. Eur J Heart Failure 2012; 14: 82-90.

8 Guazzi M, Vicenzi M, Arena R, Guazzi MD. PDE5 inhibition with sildenafil improves left ventricular diastolic function, cardiac geometry, and clinical status in patients with stable systolic heart failure: results of a 1-year, prospective, randomized, placebo-controlled study. Circ Heart Failure 2011; 4: 8-17.

9 Lewis GD, Shah R, Shahzad K, Camuso JM, Pappagianopoulos PP, Hung $J$, et al. Sildenafil improves exercise capacity and quality of life in patients with systolic heart failure and secondary pulmonary hypertension. Circulation 2007; 116: 1555-62.

10 Andersen MJ, Ersboll M, Axelsson A, Gustafsson F, Hassager C, Kober $\mathrm{L}$, et al. Sildenafil and diastolic dysfunction after acute myocardial infarction in patients with preserved ejection fraction: the Sildenafil and Diastolic Dysfunction After Acute Myocardial Infarction (SIDAMI) trial. Circulation 2013; 127: 1200-8.

11 Redfield MM, Chen HH, Borlaug BA, Semigran MJ, Lee KL, Lewis G, et al. Effect of phosphodiesterase-5 inhibition on exercise capacity and clinical status in heart failure with preserved ejection fraction: a randomized clinical trial. JAMA 2013; 309: 1268-77.

12 Giannetta E, Feola T, Gianfrilli D, Pofi R, Dall'Armi V, Badagliacca R, et al. Is chronic inhibition of phosphodiesterase type 5 cardioprotective and safe? A meta-analysis of randomized controlled trials. BMC Med 2014; $12: 185$.

13 Ferrantini C, Crocini C, Coppini R, Vanzi F, Tesi C, Cerbai E, et al. The transverse-axial tubular system of cardiomyocytes. Cell Mol Life Sci 2013; 70: 4695-710.

14 Heinzel FR, Bito V, Biesmans L, Wu M, Detre E, von Wegner F, et al. Remodeling of T-tubules and reduced synchrony of $\mathrm{Ca}^{2+}$ release in myocytes from chronically ischemic myocardium. Circ Res 2008; 102 : 338-46.

15 Louch WE, Mork HK, Sexton J, Stromme TA, Laake P, Sjaastad I, et al. T-tubule disorganization and reduced synchrony of $\mathrm{Ca}^{2+}$ release in murine cardiomyocytes following myocardial infarction. J Physiol 2006; 574: 519-33.

16 Lyon AR, MacLeod KT, Zhang Y, Garcia E, Kanda GK, Lab MJ, et al. Loss of T-tubules and other changes to surface topography in ventricular myocytes from failing human and rat heart. Proc Natl Acad Sci U S A 2009; 106: 6854-9.

17 Wei S, Guo A, Chen B, Kutschke W, Xie YP, Zimmerman K, et al. T-tubule remodeling during transition from hypertrophy to heart failure. Circ Res 2010; 107: 520-31.

18 Xie YP, Chen B, Sanders P, Guo A, Li Y, Zimmerman K, et al. Sildenafil prevents and reverses transverse-tubule remodeling and $\mathrm{Ca}^{2+}$ handling dysfunction in right ventricle failure induced by pulmonary artery hypertension. Hypertension 2012; 59: 355-62.

19 Song LS, Sobie EA, McCulle S, Lederer WJ, Balke CW, Cheng H. Orphaned ryanodine receptors in the failing heart. Proc Natl Acad Sci U S A 2006; 103: 4305-10.

20 Crossman DJ, Ruygrok PN, Soeller C, Cannell MB. Changes in the organization of excitation-contraction coupling structures in failing human heart. PloS One 2011; 6: e17901.

21 Guo A, Zhang C, Wei S, Chen B, Song LS. Emerging mechanisms of T-tubule remodelling in heart failure. Cardiovasc Res 2013; 98: 204-15.

22 Sacconi L, Ferrantini C, Lotti J, Coppini R, Yan P, Loew LM, et al. Action potential propagation in transverse-axial tubular system is impaired in heart failure. Proc Natl Acad Sci U S A 2012; 109: 5815-9.

23 Crocini C, Coppini R, Ferrantini C, Yan P, Loew LM, Tesi C, et al. Defects in T-tubular electrical activity underlie local alterations of calcium release in heart failure. Proc Natl Acad Sci U S A 2014; 111: 15196-201.

24 Crocini C, Ferrantini C, Scardigli M, Coppini R, Mazzoni L, Lazzeri E, et al. Novel insights on the relationship between T-tubular defects and contractile dysfunction in a mouse model of hypertrophic cardiomyopathy. J Mol Cell Cardiol 2016; 91: 42-51.

25 Rockman HA, Ross RS, Harris AN, Knowlton KU, Steinhelper ME, Field $\mathrm{L}$, et al. Segregation of atrial-specific and inducible expression of an atrial natriuretic factor transgene in an in vivo murine model of cardiac hypertrophy. Proc Natl Acad Sci U S A 1991; 88: 8277-81.

26 Hill JA, Karimi M, Kutschke W, Davisson RL, Zimmerman K, Wang Z, et al. Cardiac hypertrophy is not a required compensatory response to short-term pressure overload. Circulation 2000; 101: 2863-9.

27 Schiller NB, Acquatella H, Ports TA, Drew D, Goerke J, Ringertz H, et al. Left ventricular volume from paired biplane two-dimensional echocardiography. Circulation 1979; 60: 547-55.

28 Guo A, Zhang X, lyer VR, Chen B, Zhang C, Kutschke WJ, et al. Overexpression of junctophilin-2 does not enhance baseline function but attenuates heart failure development after cardiac stress. Proc Natl Acad Sci U S A 2014; 111: 12240-5.

29 Louch WE, Bito V, Heinzel FR, Macianskiene R, Vanhaecke J, Flameng $\mathrm{W}$, et al. Reduced synchrony of $\mathrm{Ca}^{2+}$ release with loss of T-tubules-a comparison to $\mathrm{Ca}^{2+}$ release in human failing cardiomyocytes. Cardiovasc Res 2004; 62: 63-73.

30 Eder E, Eickels Mv, Frantz S, Völker K, Baba HA, Kuhn M. Chronic inhibition of cyclic GMP phosphodiesterase $5 \mathrm{~A}$ may promote pressure overload-induced chamber dilatation in mice. BMC Pharmacol 2007; 7 (Suppl 1): 1-2.

31 Wu CY, Jia Z, Wang W, Ballou LM, Jiang YP, Chen B, et al. PI3Ks maintain the structural integrity of T-tubules in cardiac myocytes. PloS One 2011; 6: e24404.

32 Pokreisz P, Vandenwijngaert S, Bito V, Van den Bergh A, Lenaerts I, Busch C, et al. Ventricular phosphodiesterase-5 expression is increased in patients with advanced heart failure and contributes to adverse ventricular remodeling after myocardial infarction in mice. Circulation 2009; 119: 408-16.

33 Nagendran J, Archer SL, Soliman D, Gurtu V, Moudgil R, Haromy A, et al. Phosphodiesterase type 5 is highly expressed in the hypertrophied human right ventricle, and acute inhibition of phosphodiesterase type 5 improves contractility. Circulation 2007; 116: 238-48.

34 Lee E, Marcucci M, Daniell L, Pypaert M, Weisz OA, Ochoa GC, et al. Amphiphysin 2 (Bin1) and T-tubule biogenesis in muscle. Science 2002; 297: 1193-6.

35 Hong TT, Smyth JW, Gao D, Chu KY, Vogan JM, Fong TS, et al. BIN1 localizes the L-type calcium channel to cardiac T-tubules. PLoS Biol 2010; 8: e1000312. 
36 Hong T, Yang $\mathrm{H}$, Zhang SS, Cho HC, Kalashnikova M, Sun B, et al. Cardiac BIN1 folds T-tubule membrane, controlling ion flux and limiting arrhythmia. Nat Med 2014; 20: 624-32.

37 Chen B, Guo A, Zhang C, Chen R, Zhu Y, Hong J, et al. Critical roles of junctophilin-2 in T-tubule and excitation-contraction coupling maturation during postnatal development. Cardiovasc Res 2013; 100: 54-62.

38 Reynolds JO, Chiang DY, Wang W, Beavers DL, Dixit SS, Skapura DG, et al. Junctophilin-2 is necessary for T-tubule maturation during mouse heart development. Cardiovasc Res 2013; 100: 44-53.

39 Hong TT, Smyth JW, Chu KY, Vogan JM, Fong TS, Jensen BC, et al.
BIN1 is reduced and Cav1.2 trafficking is impaired in human failing cardiomyocytes. Heart Rhythm 2012; 9: 812-20.

40 Takimoto E, Koitabashi N, Hsu S, Ketner EA, Zhang M, Nagayama T, et al. Regulator of $\mathrm{G}$ protein signaling 2 mediates cardiac compensation to pressure overload and antihypertrophic effects of PDE5 inhibition in mice. J Clin Invest 2009; 119: 408-20.

41 Wu CY, Chen B, Jiang YP, Jia Z, Martin DW, Liu S, et al. Calpaindependent cleavage of junctophilin-2 and T-tubule remodeling in a mouse model of reversible heart failure. J Am Heart Assoc 2014; 3: e000527. 